Supporting information

\title{
Xylose-rich Horse Manure Hydrolysate as the Sole Carbon Source for Bacterial Production of Polyhydroxy Butyrate (PHB) Using Engineered E. coli
}

Hooman Torabi†, Imann Mosleh†, Seyed Mohammad Davachi†, Maryam Davaritouchaee ${ }^{\dagger}$, Alireza

Abbaspourrad $t^{*}$

†Department of Food Science, College of Agriculture \& Life Sciences, Cornell University, Stocking Hall, Ithaca, New York, 14853, United States

*Corresponding Author. Email address: Alireza@cornell.edu (A. Abbaspourrad)

Number of Pages: 7

Number of Figures: 4

Number of Tables: 1

Materials (page S2)

Plasmid construction (page S2)

phaCAB plasmid sequence (page S3)

Chemical structure of yielded PHB from 2\% D-HMH (page S5)

Thermal analysis (page S6) 


\section{Materials}

Analytical grade xylose, arabinose, glucose, isopropyl- $\beta$ - D-thiogalactoside (IPTG), furfural, and hydroxy methyl furfural (HMF) were purchased from Sigma-Aldrich. Reagent grade xylose and arabinose were purchased from Sigma-Aldrich. All other chemicals and reagents used in this study were analytical or reagent grade available in our lab stock. In all experiments, ultra-pure milli-Q water $(18 \mathrm{M} \Omega)$ was used.

\section{Plasmid construction}

Escherichia coli BL21 (DE3) competent cells (New England BioLabs ${ }^{\circledR}$ ) were used as the host strain for PHB production. A fragment containing phaCAB operon consisting of phaA (3ketothiolase), phaB (acetoacetyl-CoA reductase), and phaC (PHA synthase) genes with their ribosomal binding sites, amplified from the R. eutropha $\mathrm{H} 16$ genomic DNA was designed according to the codon preferences of $E$. coli. The fragment was then inserted into pUC57 plasmid cloning vector isolated from $E$. coli strain $\mathrm{DH} 5 \alpha$ with an ampicillin resistance marker and T7 promoter by Biomatik Corporation (Cambridge, Canada). Detailed information of the plasmid is provided in supplementary information. E. coli competent cells were transformed with the plasmid using heat shock according to manufacturer's protocol. Cells were then plated on Luria-Bertani (LB) agar media selection plate containing $50 \mu \mathrm{g} / \mathrm{mL}$ ampicillin and were incubated overnight at $37^{\circ} \mathrm{C}$. The success of process was confirmed by formation of colonies on an agar plate. 


\section{phaCAB plasmid Sequence:}

aattaatacgactcactataggggaattgtgagcggataacaattcccctctagaaataattttgtttaactttaagaaggagatatacatatggctact gggaaaggagccgccgccagcacccaggaaggcaaaagccaaccatttaaagtgaccccaggcccgttcgatcctgccacctggctggaatggtca cgtcaatggcaaggtacggagggcaacggccatgcggcagcgtccggcatccctgggcttgatgcactggctggtgttaaaattgcgcctgcccaatt gggagacatccaacagcgttatatgaaagatttttcggcgctgtggcaggcgatggcggaaggtaaagcagaggcgacgggaccgctgcatgaccg tcgctttgctggcgacgcctggcgtaccaatttgccctatcgttttgcggcagccttctacctgctgaacgctcgtgccctgactgagctggcagatgctg tcgaagcggatgccaaaacccgccaacgcatccgttttgccatctcacagtgggtcgacgcaatgtcaccggccaacttcctggccaccaatccggaa gcacagcgcctcctgattgaaagcggcggggaatcgctgcgcgccggcgttcgtaacatgatggaagatctgacccgtggtaagatttcgcagacgg atgagtcggccttcgaggtgggtcgcaatgttgcagtgacagaaggcgcggtggtgtttgagaacgaatactttcaacttctccagtataaaccactga cggataaagtgcatgcgcgcccattgttaatggttcccccgtgcattaataaatactatatcctggatcttcagccggaaagctccttagtgcgccacgt ggtggaacaggggcatactgtgtttttagttagctggcgcaatccagatgcctcaatggccggcagtacctgggatgactacattgaacacgcggctat tcgcgcgatcgaagtggcacgcgacatctcaggtcaggacaagattaacgtactcggcttttgcgtcggtggcactatcgtgagcaccgctctggcggt actggctgcgcgtggtgaacaccccgcagcgtcggttacgcttctgaccacactgttggatttcgccgacactggcatcttggatgtatttgtcgatgag ggccacgttcagctgcgcgaggccactctgggtggcggcgccggcgcaccgtgcgctctgctgcgcggtctggagctcgcgaatacttttctttcttac gcccgaacgatcttgtctggaattacgtagttgacaactatctcaaaggtaacacgcccgtgccgtttgatttattattctggaatggggatgcgaccaa cctgccgggtccttggtattgttggtatctccgtcatacatacttacagaatgaattaaaggtcccaggtaaacttactgtgtgtggtgtcccggtagatc tggcgagtatcgacgtgccgacctacatttatgggagtcgtgaagatcatattgtgccgtggaccgccgcgtacgcttctaccgcgcttctggcaaaca aattacgtttcgttctgggtgccagcggccacattgcgggtgttattaatcctccggccaaaaataaacgctcgcattggacaaacgacgccttgccgg agagccctcagcagtggttggcgggtgccattgagcaccatggaagttggtggccggactggacagcctggctggcgggccaagcaggcgcaaaac gcgctgcgccagccaactatggcaacgcgcgctaccgcgcgattgaacccgcgcctggccgctatgtaaaagcaaaagcgtgacgcttgcatgagtg ccggcgtgcgtcatgcacggcgccggcaggcctgcacgttccctcccgtttccattgaaaggactacacaatgaccgatgttgttatcgtcagtgccgc gcgcaccgccgtgggtaaatttggcggcagtctggcgaaaatcccggcgccagaattgggcgcggtggtaatcaaagcggccctggaacgcgccggt gtgaaacctgagcaggtgagcgaagtaattatgggtcaggttctgaccgcgggcagcgggcaaaatccggcgcgtcaggctgcgattaaggcaggc cttccggcgatggtgcccgcgatgaccattaacaaagtatgcggatcgggactgaaagcagtgatgctggccgctaacgccattatggcaggcgacg cggaaattgtggtcgctggcggacaggagaatatgtcagccgcgcctcatgtgctgccgggtagccgcgacggctttcgcatgggtgatgcaaaactg gtcgatactatgattgttgacggcttatgggatgtgtacaaccagtaccacatgggcatcaccgccgagaacgtggccaaggaatacggcatcacacg cgaggcgcaggatgagttcgccgtcggctcgcagaacaaggccgaagccgcgcagaaggccggcatgtttgacgaagagatcgtcccggtgctgat cccgcagcgcaagggcgacccggtggccttcaagaccgacgagttcgtgcgccagggcgccacgctggacagcatgtccggcctcaagcccgccttc gacaaggccggcacggtgaccgcggccaacgcctcgggcctgaacgacggcgccgccgcggtggtggtgatgtcggcggccaaggccaaggaact gggcctgaccccgctggccacgatcaagagctatgccaacgccggtgtcgatcccaaggtgatgggcatgggcccggtgccggcctccaagcgcgcc ctgtcgcgcgccgagtggaccccgcaagacctggacctgatggagatcaacgaggcctttgccgcgcaggcgctggcggtgcaccagcagatgggct gggacacctccaaggtcaatgtgaacggcggcgccatcgccatcggccacccgatcggcgcgtcgggctgccgtatcctggtgacgctgctgcacga 
gatgaagcgccgtgacgcgaagaagggcctggcctcgctgtgcatcggcggcggcatgggcgtggcgctggcagtcgagcgcaaataaggaaggg gttttccggggccgcgcgcggttggcgcggacccggcgacgataacgaagccaatcaaggagtggacatgactcagcgcattgcgtatgtgaccggc ggcatgggtggtatcggaaccgccatttgccagcggctggccaaggatggctttcgtgtggtggccggttgcggccccaactcgccgcgccgcgaaaa gtggctggagcagcagaaggccctgggcttcgatttcattgcctcggaaggcaatgtggctgactgggactcgaccaagaccgcattcgacaaggtca agtccgaggtcggcgaggttgatgtgctgatcaacaacgccggtatcacccgcgacgtggtgttccgcaagatgacccgcgccgactgggatgcggtg atcgacaccaacctgacctcgctgttcaacgtcaccaagcaggtgatcgacggcatggccgaccgtggctggggccgcatcgtcaacatctcgtcggt gaacgggcagaagggccagttcggccagaccaactactccaccgccaaggccggcctgcatggcttcaccatggcactggcgcaggaagtggcgac caagggcgtgaccgtcaacacggtctctccgggctatatcgccaccgacatggtcaaggcgatccgccaggacgtgctcgacaagatcgtcgcgacg atcccggtcaagcgcctgggcctgccggaagagatcgcctcgatctgcgcctggttgtcgtcggaggagtccggtttctcgaccggcgccgacttctcg ctcaacggcggcctgcatatgggctgacctgc

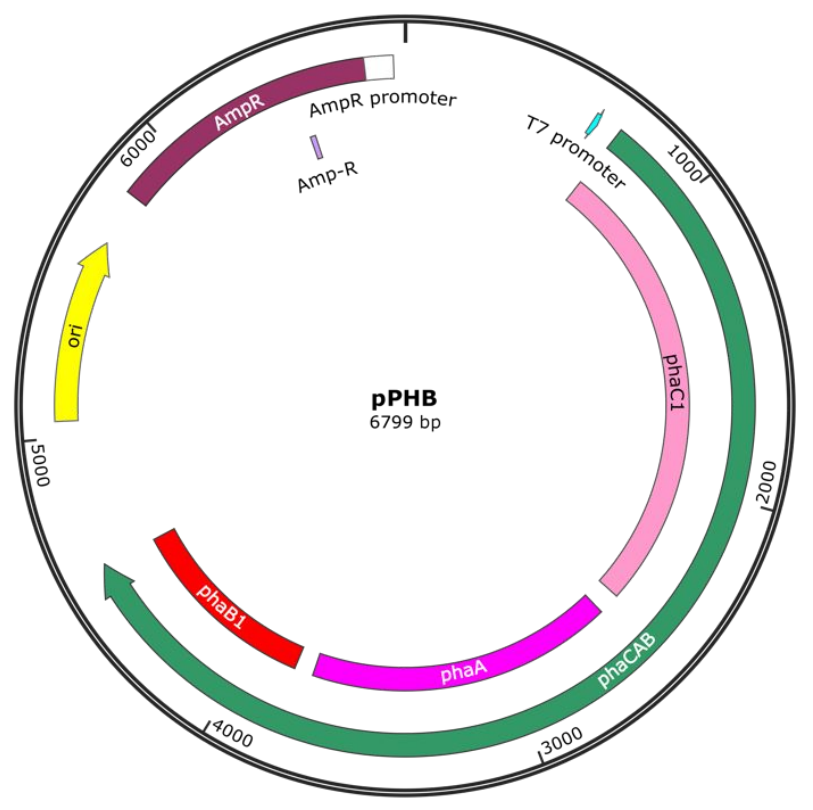

\section{0k bp $8 \mathrm{kbp}$ $5 \mathrm{kbp}$ $2 \mathrm{kbp}$ \\ $1.5 \mathrm{k} \mathrm{bp}$ $1 \mathrm{k} \mathrm{bp}$ $750 \mathrm{bp}$ 500 bp $250 \mathrm{bp}$}

Figure S1: (left) pPHB plasmid map harboring phaCAB operon and (right) agarose electrophoresis gel run for constructed plasmid. 


\section{Chemical structure of the PHB yielded from 2\% D-HMH}

The FT-IR spectra (Figure S2) exhibited characteristic bands for the ester bonds which are visible at 1028 $\mathrm{cm}^{-1}$ (O-C-C stretch), $1260 \mathrm{~cm}^{-1}$ (C-C-O stretch), and $1724 \mathrm{~cm}^{-1}$ (C=O stretch). In addition, the bands at $1380 \mathrm{~cm}^{-1}, 1458 \mathrm{~cm}^{-1}$, and $2922 \mathrm{~cm}^{-1}$ indicated $\mathrm{CH}_{3},-\mathrm{CH}_{2}$, and $-\mathrm{CH}$ groups, respectively.

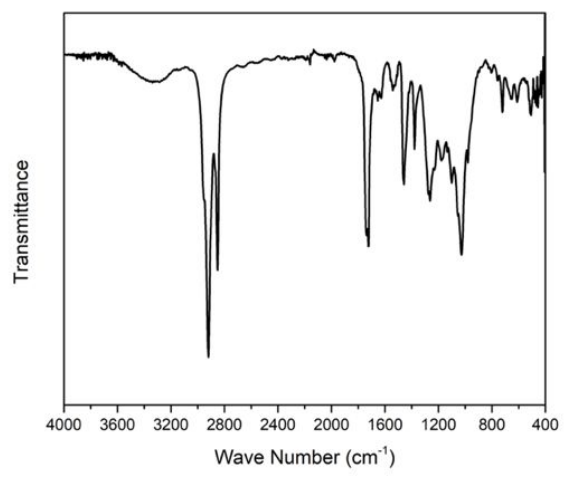

Figure S2: FT-IR spectrum of PHB derived from cells on D-HMH after $72 \mathrm{~h}$.

${ }^{1} \mathrm{HNMR}\left(500 \mathrm{MHz}, \mathrm{CDCl}_{3}\right)$ spectra also confirms the proposed structure of the isolated polymer (PHB) as $\delta(\mathrm{ppm}): 7.26$ (Chloroform-d), $5.20(\mathrm{~m}, 1 \mathrm{H}), 2.51(\mathrm{~d}, 1 \mathrm{H}), 2.41(\mathrm{~d}, 1 \mathrm{H})$, and $1.21(\mathrm{~d}, 3 \mathrm{H})$.

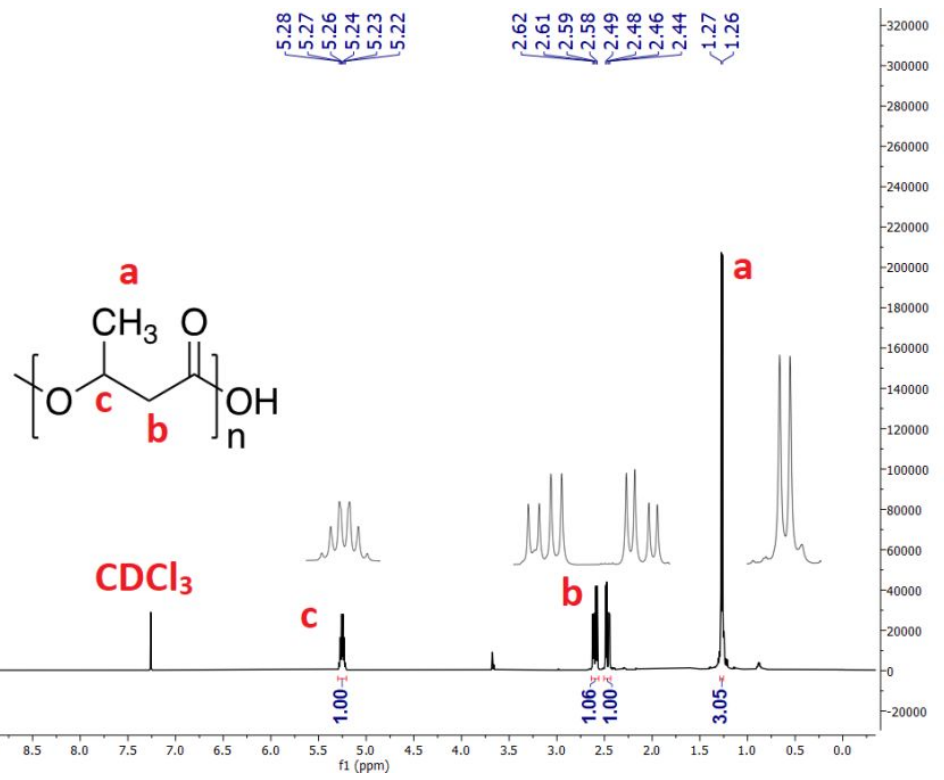

Figure S3: ${ }^{1} \mathrm{H} N \mathrm{NR}$ spectrum in $\mathrm{CDCl}_{3}$ of $\mathrm{PHB}$ derived from cells on $\mathrm{D}-\mathrm{HMH}$ after $72 \mathrm{~h}$ 


\section{Thermal analysis}

The DSC tests were performed at a heating rate of $10^{\circ} \mathrm{C} / \mathrm{min}$ in a nitrogen atmosphere at temperatures ranging from -50 to $275^{\circ} \mathrm{C}$. The samples were heated from 25 to $150^{\circ} \mathrm{C}$ and then cooled to $-50{ }^{\circ} \mathrm{C}$, to erase the thermal history. At the final heating stage, the samples were heated from -40 to $275^{\circ} \mathrm{C}$ and the resulting thermographs are depicted in Figure S4. Upon an increase in incubation time, all the samples demonstrated an increase in molecular weights, regardless of feedstock. Therefore, $T_{g}, T_{m}$, and $T_{d}$ have all shifted to higher temperatures (Table S1); due to the increase in molecular interactions between the chains and a decrease in chain mobility. The melting enthalpy was increased from 46.2 $\mathrm{J} / \mathrm{mol}$ after the $48 \mathrm{~h}$ incubation to $68.4 \mathrm{~J} / \mathrm{mol}$ after $72 \mathrm{~h}$ for the PHB yielded from D-HMH. A similar change was also observed for the control group with an increase from $49.9 \mathrm{~J} / \mathrm{mol}$ after $48 \mathrm{~h}$ to 71.4 $\mathrm{J} / \mathrm{mol}$ after $72 \mathrm{~h}$.

Table S1 Thermal properties of the extracted PHB

\begin{tabular}{ccccccc}
\hline Carbon source & Incubation time $(\mathrm{h})$ & $\mathrm{T}_{\mathrm{g}}\left({ }^{\circ} \mathrm{C}\right)$ & $\mathrm{T}_{\mathrm{m}}\left({ }^{\circ} \mathrm{C}\right)$ & $\mathrm{T}_{\mathrm{d}}\left({ }^{\circ} \mathrm{C}\right)$ & $\Delta \mathrm{H}_{\mathrm{m}}(\mathrm{J} / \mathrm{g})$ & ${ }^{\mathrm{a}} \mathbf{X}_{\mathrm{c}}(\%)$ \\
\hline D-HMH & 48 & 3.5 & 171.7 & 191.0 & 46.2 & 31.6 \\
& 72 & 4.3 & 177.0 & 204.6 & 68.4 & 44.8 \\
\hline Xylose & 48 & 3.6 & 172.7 & 193.9 & 49.9 & 34.2 \\
(control) & 72 & 5.9 & 180.2 & 213.9 & 71.4 & 49.6 \\
\hline Arabinose & & & & & & \\
\hline
\end{tabular}




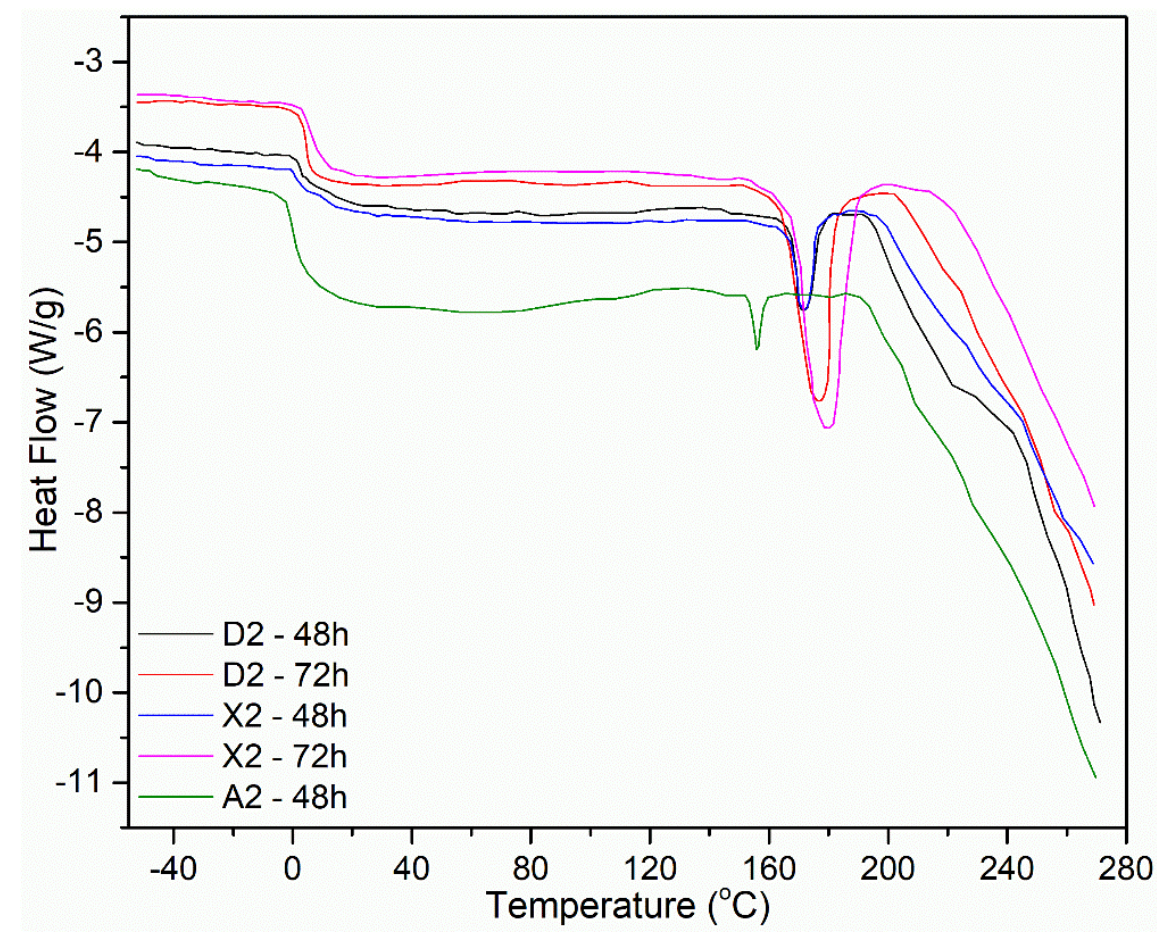

Figure S4: DSC thermograms of produced PHB using D2 (2\% W/V D-HMH), X2 (2\% W/V xylose), and A2 (2\% W/V arabinose) as carbon source at designated timeframes 